\title{
EDITORIAL
}

\section{The role of stress in rheumatic diseases}

\author{
Afton L Hassett* and Daniel J Clauw \\ See related research by de Brouwer et al., http://arthritis-research.com/content/12/3/R89
}

\begin{abstract}
Rheumatology patients frequently note the occurrence of stressful or traumatic life events prior to the onset of their illness and/or a relationship between stress and disease flares. For our patients, identifying causal events could represent an effort to give meaning to a chronic and often disabling disease, while noting a link between stress and flares may proffer a sense of control. Whatever purpose the report of stress as an etiological or maintaining factor may serve, the science exploring a causal relationship between stress and autoimmune disease onset and course is expanding. Moreover, stress can also induce symptoms such as pain via nonimmunological mechanisms.
\end{abstract}

In the present issue of Arthritis Research \& Therapy, de Brouwer and colleagues review the literature pertaining to experimental studies targeting acute-phase reactivity in the stress-response systems of patients with rheumatoid arthritis (RA) and systemic lupus erythematosus (SLE) [1]. The authors included only studies employing experimental stressors (psychosocial, cognitive, exercise, and sensory/pain induction) to evaluate physiological responses at three levels - the autonomic nervous system (ANS), the hypothalamic-pituitary-adrenal (HPA) axis, and the immune system - in patients with RA and SLE.

Sixteen studies were identified [1]. The authors found inconsistent results regarding experimentally induced stress and the ANS and HPA axis baseline levels and reactivity, but found some evidence for alterations in immune functioning in patients compared with controls. They noted that the most consistent finding in response to experimentally induced stress was an increase in the number of natural killer cells, but this may not be surprising because natural killer cell trafficking is very

*Correspondence: afton@med.umich.edu

Chronic Pain \& Fatigue Research Center, Department of Anesthesiology, University of Michigan Medical School, 24 Frank Lloyd Wright Drive, PO Box 385, Lobby M, Ann Arbor, Michigan 48106, USA sensitive to stress hormones such as catecholamines. The authors note, however, that many of the studies possessed methodological problems of their own. Most studies were underpowered (that is, small sample sizes) and some failed to control for potential confounders such as medication use, age, sex, psychiatric comorbidity, stress coping/appraisal and abuse history. In patients with rheumatologic illness, the presence of depression and a history of abuse are relatively common and have been associated with alterations in the stress-response and immune systems $[2,3]$.

The results from the present review of the effects of stress in RA and SLE suggest that the findings in these disorders are congruent with a broader literature including both animal models and clinical studies of other rheumatic disorders. A number of different types of stress have been shown to induce arthritis in animal models [4]; however, such a relationship in humans is more tenuous.

Most studies are limited by the use of cross-sectional designs and the pitfalls associated with self-report retrospective data, but their findings are still of interest. For example, a study of Vietnam combat veterans with current post-traumatic stress disorder $(n=2,490)$ found that they were at increased risk for autoimmune diseases $(16.7 \%, 95 \%$ confidence interval $=7.9$ to $29.3 \%)$ compared with those without post-traumatic stress disorder $(6.1 \%$, $P<0.05)$ [5]. In that study, the combination of several stress-related conditions seemed to further increase this risk, with $8.1 \%$ of these male veterans with both posttraumatic stress disorder and comorbid depression, anxiety or other significant psychopathology reporting a diagnosis of RA [5].

Other studies have contemplated a role for early life stressors in increasing vulnerability to autoimmune disease. One recent study found that individuals reporting two or more traumatic childhood events were at a $100 \%$ increased risk for rheumatic diseases compared with those reporting no childhood trauma [6]. Further, a multitude of studies have described relationships between psychological stress and poor outcomes in both RA and SLE including disease flares. The mechanisms presumed to underlie these associations include stressrelated changes in functioning of the autonomic, neuroendocrine and/or immune systems. 
Work performed to examine how stress modulates symptoms, especially pain, in other nonautoimmune rheumatic conditions such as fibromyalgia might also be instructive in elucidating the role of stress in symptom expression. From a vast array of experimental studies it is reasonable to conclude that a variety of stressors may cause pain, that pain may cause stress, and, more importantly, that a simple unidirectional relationship between changes in stress-response function and pain and other symptoms probably does not exist. Imaging studies of pain processing in fibromyalgia indicate that psychological stress (that is, depression, anxiety) and pain are processed somewhat independently in the central nervous system [7]. Supporting this conclusion are the clinical data indicating that drugs acting as both antidepressants and analgesics (for example, tricyclics or serotonin-norepinephrine reuptake inhibitors) are equally effective analgesics in chronic pain conditions in patients with and without depression [8]. The lack of direct overlap in the central processing of stress and pain suggests that the degree to which stress influences pain, and vice versa, may be moderated by individual factors such as cognitions, coping/appraisal and social support $[9,10]$.

In fibromyalgia, as well as in autoimmune disorders, symptom expression is likely determined to a similar extent by genetic and environmental factors. Although there have been efforts to link pain and subtle abnormalities in ANS and HPA system functioning seen in individuals with fibromyalgia and other chronic pain syndromes, newer data suggest much more complex interrelationships between these systems. Observable changes in the ANS or HPA axis tone in some individuals may represent a baseline diathesis or risk factor for the subsequent development of chronic pain, or the changes may be due to pain itself or to the indirect effects of pain such as deconditioning secondary to decreased exercise.

In summary, when our patients say that stress worsens their disease, they may be correct. Although the study of stress is fraught with problems, there are clearly both immune mechanisms (that is, psychoneuroimmunology) and nonimmune mechanisms (for example, mechanisms operative in conditions such as fibromyalgia, either alone or comorbid with autoimmune disorders) that may be responsible for increased disease activity and/or symptom expression during periods of stress.

\section{Abbreviations}

ANS, autonomic nervous system; HPA, hypothalamic-pituitary-adrenal; RA, rheumatoid arthritis; SLE, systemic lupus erythematosus.

\section{Competing interests}

The authors declare that they have no competing interests.

\section{Published: 7 June 2010}

\section{References}

1. de Brouwer SJM, Kraaimaat FW, Sweep FCGJ, Creemers MCW, Radstake TRDJ, van Laarhoven AIM, van Riel PLCM, Evers AWM: Experimental stress in inflammatory rheumatic diseases: a review of psychophysiological stress responses. Arthritis Therapy Res 2010, 12:R89.

2. Kojima M, Kojima T, Suzuki S, Oguchi T, Oba M, Tsuchiya H, Sugiura F, Kanayama Y, Furukawa TA, Tokudome S, Ishiguro N: Depression, inflammation, and pain in patients with rheumatoid arthritis. Arthritis Rheum 2009, 61:1018-1024.

3. McLean SA, Williams DA, Stein PK, Harris RE, Lyden AK, Whalen G, Park KM, Liberzon I, Sen A, Gracely RH, Baraniuk JN, Clauw DJ: Cerebrospinal fluid corticotropin-releasing factor concentration is associated with pain but not fatigue symptoms in patients with fibromyalgia. Neuropsychopharmacology 2006, 31:2776-2782.

4. Harbuz MS, Richards L, Chover-Gonzalez AJ, Marti-Sistac O, Jessop DS: Stress in autoimmune disease models. Ann NY Acad Sci 2006, 1069:51-61.

5. Boscarino JA: Posttraumatic stress disorder and physical illness. Results from clinical and epidemiologic studies. Ann NY Acad Sci 2004, 1032:141-153.

6. Dube SR, Fairweather D, Pearson WS, Felitti VJ, Anda RF, Croft JB: Cumulative childhood stress and autoimmune disease in adults. Psychosom Med 2009, 71:243-250.

7. Giesecke T, Gracely RH, Williams DA, Geisser ME, Petzke FW, Clauw DJ: The relationship between depression, clinical pain, and experimental pain in a chronic pain cohort. Arthritis Rheum 2005, 52:1577-1584

8. Arnold LM: Duloxetine and other antidepressants in the treatment of patients with fibromyalgia. Pain Med 2007, 8(Suppl 2):S63-S74.

9. Gracely RH, Geisser ME, Giesecke T, Grant MA, Petzke F, Williams DA, Clauw DJ: Pain catastrophizing and neural responses to pain among persons with fibromyalgia. Brain 2004, 127(Pt 4):835-843.

10. Montoya P, Larbig W, Braun C, PreissI H, Birbaumer N: Influence of social support and emotional context on pain processing and magnetic brain responses in fibromyalgia. Arthritis Rheum 2004, 50:4035-4044

doi:10.1186/ar3024

Cite this article as: Hassett AL, Clauw DJ: The role of stress in rheumatic diseases. Arthritis Research \& Therapy 2010, 12:123. 